\title{
The fate of hints: updated global analysis of three-flavor neutrino oscillations
}

\author{
Ivan Esteban, ${ }^{a}$ M.C. Gonzalez-Garcia, ${ }^{a, b, c}$ Michele Maltoni, ${ }^{d}$ Thomas Schwetz ${ }^{e}$ \\ and Albert Zhou ${ }^{e}$ \\ ${ }^{a}$ Departament de Fisica Quàntica i Astrofísica and Institut de Ciencies del Cosmos, \\ Universitat de Barcelona, Diagonal 647, E-08028 Barcelona, Spain \\ ${ }^{b}$ Institució Catalana de Recerca i Estudis Avançats (ICREA), \\ Pg. Lluis Companys 23, 08010 Barcelona, Spain. \\ ${ }^{c}$ C.N. Yang Institute for Theoretical Physics, State University of New York at Stony Brook, \\ Stony Brook, NY 11794-3840, U.S.A. \\ ${ }^{d}$ Instituto de Física Teórica UAM/CSIC, Calle de Nicolás Cabrera 13-15, \\ Universidad Autónoma de Madrid, Cantoblanco, E-28049 Madrid, Spain \\ ${ }^{e}$ Institut für Astroteilchenphysik, Karlsruher Institut für Technologie (KIT), \\ D-76021 Karlsruhe, Germany \\ E-mail: ivan.esteban@fqa.ub.edu, \\ maria.gonzalez-garcia@stonybrook.edu, michele.maltoni@csic.es, \\ schwetz@kit.edu, albert.zhou@kit.edu
}

ABSTRACT: Our herein described combined analysis of the latest neutrino oscillation data presented at the Neutrino2020 conference shows that previous hints for the neutrino mass ordering have significantly decreased, and normal ordering (NO) is favored only at the $1.6 \sigma$ level. Combined with the $\chi^{2}$ map provided by Super-Kamiokande for their atmospheric neutrino data analysis the hint for $\mathrm{NO}$ is at $2.7 \sigma$. The $\mathrm{CP}$ conserving value $\delta_{\mathrm{CP}}=180^{\circ}$ is within $0.6 \sigma$ of the global best fit point. Only if we restrict to inverted mass ordering, $\mathrm{CP}$ violation is favored at the $\sim 3 \sigma$ level. We discuss the origin of these results - which are driven by the new data from the T2K and NOvA long-baseline experiments - , and the relevance of the LBL-reactor oscillation frequency complementarity. The previous $2.2 \sigma$ tension in $\Delta m^{2}{ }_{21}$ preferred by KamLAND and solar experiments is also reduced to the $1.1 \sigma$ level after the inclusion of the latest Super-Kamiokande solar neutrino results. Finally we present updated allowed ranges for the oscillation parameters and for the leptonic Jarlskog determinant from the global analysis.

Keywords: Neutrino Physics, CP violation, Solar and Atmospheric Neutrinos

ARXIV EPRINT: 2007.14792 


\section{Contents}

1 Introduction 1

2 Fading hints for CP violation and neutrino mass ordering 2

2.1 T2K and NOvA updates 2

$\begin{array}{lll}2.2 & \text { Accelerator versus reactor } & 6\end{array}$

2.3 Consistency between T2K, NOvA and reactors 8

$\begin{array}{lll}3 & \text { Resolved tension in the solar sector } & 9\end{array}$

4 Global fit results 10

$\begin{array}{llr}5 & \text { Summary } & 15\end{array}$

$\begin{array}{ll}\text { A List of data used in the analysis } & 17\end{array}$

\section{Introduction}

Global fits to neutrino oscillation data in the last several years have shown persistent hints for the normal neutrino mass ordering and values of the $\mathrm{CP}$ phase $\delta_{\mathrm{CP}}$ around maximal $\mathrm{CP}$ violation [1-6]. In this article we are going to re-assess the status of those hints in light of the new data released at the Neutrino2020 conference, in particular by the T2K $[7,8]$ and NOvA $[9,10]$ long-baseline (LBL) experiments. As we are going to discuss in detail, the hints have mostly disappeared or are significantly decreased: both neutrino mass orderings provide fits of comparable quality to the global data from accelerator and reactor experiments, and the $\mathrm{CP}$ conserving value $\delta_{\mathrm{CP}}=180^{\circ}$ is within the $1 \sigma$ allowed range.

We discuss in detail the origin of this apparent change of trends and trace back the data samples responsible for the change. We are going to compare the latest status with our pre-Neutrino2020 analysis, NuFIT 4.1, available at the NuFIT website [11]. Most relevant for mass ordering and $\mathrm{CP}$ phase are the updates of the neutrino samples for T2K [8], from 1.49 to $1.97 \times 10^{21} \mathrm{POT}$, and NOvA [10], from 0.885 to $1.36 \times 10^{21} \mathrm{POT}$. The T2K and NOvA anti-neutrino exposures are the same as used for NuFIT 4.1, but both collaborations introduced relevant changes in their analysis and hence we have adapted also our antineutrino fits correspondingly. In addition we have updated the reactor experiments DoubleChooz [12, 13] from 818/258 to 1276/587 days of far/near detector data and RENO [14, 15] from 2200 to 2908 days of exposure.

Another update concerns the solar neutrino oscillation analysis, to include the latest total energy spectrum and the day-night asymmetry of the SK4 2970-day sample presented 
at Neutrino2020 [16]. As we will show, thanks to these new data the tension on the determination of $\Delta m_{21}^{2}$ from KamLAND versus solar experiments has basically disappeared.

The outline of the paper is as follows. In section 2 we discuss the status of the neutrino mass ordering and the leptonic $\mathrm{CP}$ phase $\delta_{\mathrm{CP}}$, focusing on recent updates from T2K, NOvA, as well as the combination of LBL accelerator and reactor experiments. Despite somewhat different tendencies, we will show quantitatively that results from $\mathrm{T} 2 \mathrm{~K}$ and NOvA as well as reactors are fully statistically compatible. The status of the tension between solar and KamLAND results is presented in section 3. Section 4 contains a selection of the combined results of this global fit, NuFIT 5.0, which updates our previous analyses [1, 2, 17, 18]. In particular we present the ranges of allowed values for the oscillation parameters and of the leptonic Jarlskog determinant. ${ }^{1}$ Parametrization conventions and technical details on our global analysis can be found in ref. [2]. In particular, in what follows we use the definition

$$
\Delta m_{3 \ell}^{2} \text { with } \begin{cases}\ell=1 & \text { for } \Delta m_{3 \ell}^{2}>0: \text { normal ordering (NO), } \\ \ell=2 & \text { for } \Delta m_{3 \ell}^{2}<0: \text { inverted ordering (IO). }\end{cases}
$$

We finish by summarizing our results in section 5. A full list of the data used in this analysis is given in appendix A.

Throughout this paper we are assuming that Wilk's theorem holds to convert $\Delta \chi^{2}$ values into confidence levels and equivalent numbers of Gaussian standard deviations $(\sigma)$. The validity of this approach has been tested in previous papers by Monte Carlo simulation $[1,19]$, where we have found that the approximation works reasonably well in most cases, including also for the mass ordering.

\section{Fading hints for CP violation and neutrino mass ordering}

\section{$2.1 \quad$ T2K and NOvA updates}

We start by discussing the implications of the latest data from the T2K and NOvA longbaseline accelerator experiments, presented at the Neutrino2020 conference. ${ }^{2}$ To obtain a qualitative understanding we follow refs. $[2,19]$ and expand the oscillation probability relevant for the T2K and NOvA appearance channels in the small parameters $\sin \theta_{13}$, $\Delta m_{21}^{2} L / E_{\nu}$, and $A \equiv\left|2 E_{\nu} V / \Delta m_{3 \ell}^{2}\right|$, where $L$ is the baseline, $E_{\nu}$ the neutrino energy and $V$ the effective matter potential [21]:

$$
\begin{aligned}
& P_{\nu_{\mu} \rightarrow \nu_{e}} \approx 4 s_{13}^{2} s_{23}^{2}(1+2 o A)-C \sin \delta_{\mathrm{CP}}(1+o A), \\
& P_{\bar{\nu}_{\mu} \rightarrow \bar{\nu}_{e}} \approx 4 s_{13}^{2} s_{23}^{2}(1-2 o A)+C \sin \delta_{\mathrm{CP}}(1-o A) .
\end{aligned}
$$

with $s_{i j} \equiv \sin \theta_{i j}$ and

$$
C \equiv \frac{\Delta m_{21}^{2} L}{4 E_{\nu}} \sin 2 \theta_{12} \sin 2 \theta_{13} \sin 2 \theta_{23}, \quad o \equiv \operatorname{sgn}\left(\Delta m_{3 \ell}^{2}\right),
$$

\footnotetext{
${ }^{1}$ Additional figures, $\Delta \chi^{2}$ maps and future updates of this analysis will be made available at the NuFIT website [11].

${ }^{2}$ During the preparation of this work ref. [20] appeared presenting related partial results in qualitative agreement with some of our findings for the LBL analysis.
} 


\begin{tabular}{|c|c|c|c|c|c|c|}
\hline & \multicolumn{3}{|c|}{$\mathrm{T} 2 \mathrm{~K}(\nu)$} & \multirow[t]{2}{*}{$\mathrm{T} 2 \mathrm{~K}(\bar{\nu})$} & \multirow[t]{2}{*}{ NOvA $(\nu)$} & \multirow[t]{2}{*}{ NOvA $(\bar{\nu})$} \\
\hline & CCQE & $\mathrm{CC} 1 \pi$ & sum & & & \\
\hline $\mathcal{N}$ & 48.5 & 5 & 53.5 & 16 & 48.5 & 23 \\
\hline$N_{\text {obs }}$ & 94 & 14 & 108 & 16 & 82 & 33 \\
\hline$N_{\mathrm{obs}}-N_{\mathrm{bck}}$ & 76 & 12.1 & 88.1 & 9.8 & 55.2 & 19 \\
\hline ratio $r$ & $1.6(1.5)$ & $2.4(3.6)$ & $1.65(1.71)$ & $0.61(0.7)$ & $1.14(1.3)$ & $0.83(0.7)$ \\
\hline
\end{tabular}

Table 1. Normalization coefficients $\mathcal{N}_{\nu}$ and $\mathcal{N}_{\bar{\nu}}$ in eqs. (2.4) and (2.5) for approximations used to qualitatively describe the appearance event samples for T2K and NOvA. We also give the observed number of events, as well as the corresponding background subtracted event numbers, as reported in refs. $[8,10]$. The ratio in the last line is defined as $r=\left(N_{\text {obs }}-N_{\text {bck }}\right) / \mathcal{N}$ and numbers in brackets are the corresponding values for the data set used for NuFIT 4.1.

and we have used $\left|\Delta m_{3 \ell}^{2}\right| L / 4 E_{\nu} \approx \pi / 2$ for T2K and NOvA. At T2K, the mean neutrino energy gives $A \approx 0.05$, whereas for NOvA we find that with the empirical value of $A=0.1$ the approximation works best. Assuming that the number of observed appearance events in T2K and NOvA is approximately proportional to the oscillation probability we obtain

$$
\begin{aligned}
& N_{\nu_{e}} \approx \mathcal{N}_{\nu}\left[2 s_{23}^{2}(1+2 o A)-C^{\prime} \sin \delta_{\mathrm{CP}}(1+o A)\right], \\
& N_{\bar{\nu}_{e}} \approx \mathcal{N}_{\bar{\nu}}\left[2 s_{23}^{2}(1-2 o A)+C^{\prime} \sin \delta_{\mathrm{CP}}(1-o A)\right] .
\end{aligned}
$$

Taking all the well-determined parameters $\theta_{13}, \theta_{12}, \Delta m_{21}^{2},\left|\Delta m_{3 \ell}^{2}\right|$ at their global best fit points, we obtain numerically $C^{\prime} \approx 0.28$ with negligible dependence on $\theta_{23}$. The normalization constants $\mathcal{N}_{\nu, \bar{\nu}}$ calculated from our re-analysis of T2K and NOvA are given for the various appearance samples in table 1. Hence the expression in eqs. (2.4) and (2.5) serve well to understand the main behaviour under varying the parameters $\sin ^{2} \theta_{23}, \delta_{\mathrm{CP}}$, and the mass ordering.

In table 1 we also show the observed number of events, background subtracted events, as well as the ratio $r=\left(N_{\text {obs }}-N_{\text {bck }}\right) / \mathcal{N}_{\nu(\bar{\nu})}$. In a fit, the values of $r$ have to be accommodated by the expression in the square brackets of eqs. (2.4) and (2.5). In brackets, we give also the $r$ values for the NuFIT 4.1 data set, to illustrate the impact of the latest data.

Similar information is presented graphically in figure 1 , showing the predicted number of events for the various appearance event samples as a function of $\delta_{\mathrm{CP}}$, changing $\sin ^{2} \theta_{23}$ as well as the ordering, compared to the observed event number. Here the predictions are calculated using our experiment simulation based on fully numerical oscillation probabilities, while the general behaviour of the curves is well described by eqs. (2.4) and (2.5).

We can clearly observe a number of tendencies. T2K data has $r>1$ for neutrinos and $r<1$ for anti-neutrinos, implying that the square-bracket in $(2.4)[(2.5)]$ has to be enhanced [suppressed]. If $\theta_{13}$ is fixed as determined by reactor experiments this can be achieved by choosing $\mathrm{NO}$ and $\delta_{\mathrm{CP}} \simeq 3 \pi / 2$ (see section 2.2 for a consistent combination of reactor and LBL data). This has been the driving factor for previous hints for NO and maximal $\mathrm{CP}$ violation. We observe from the last row in table 1 that indeed this tendency has become somewhat weaker with the new data, though still clearly present. In this respect an interesting role is played by the $\mathrm{CC} 1 \pi$ event sample. A value $r=3.6$ for 

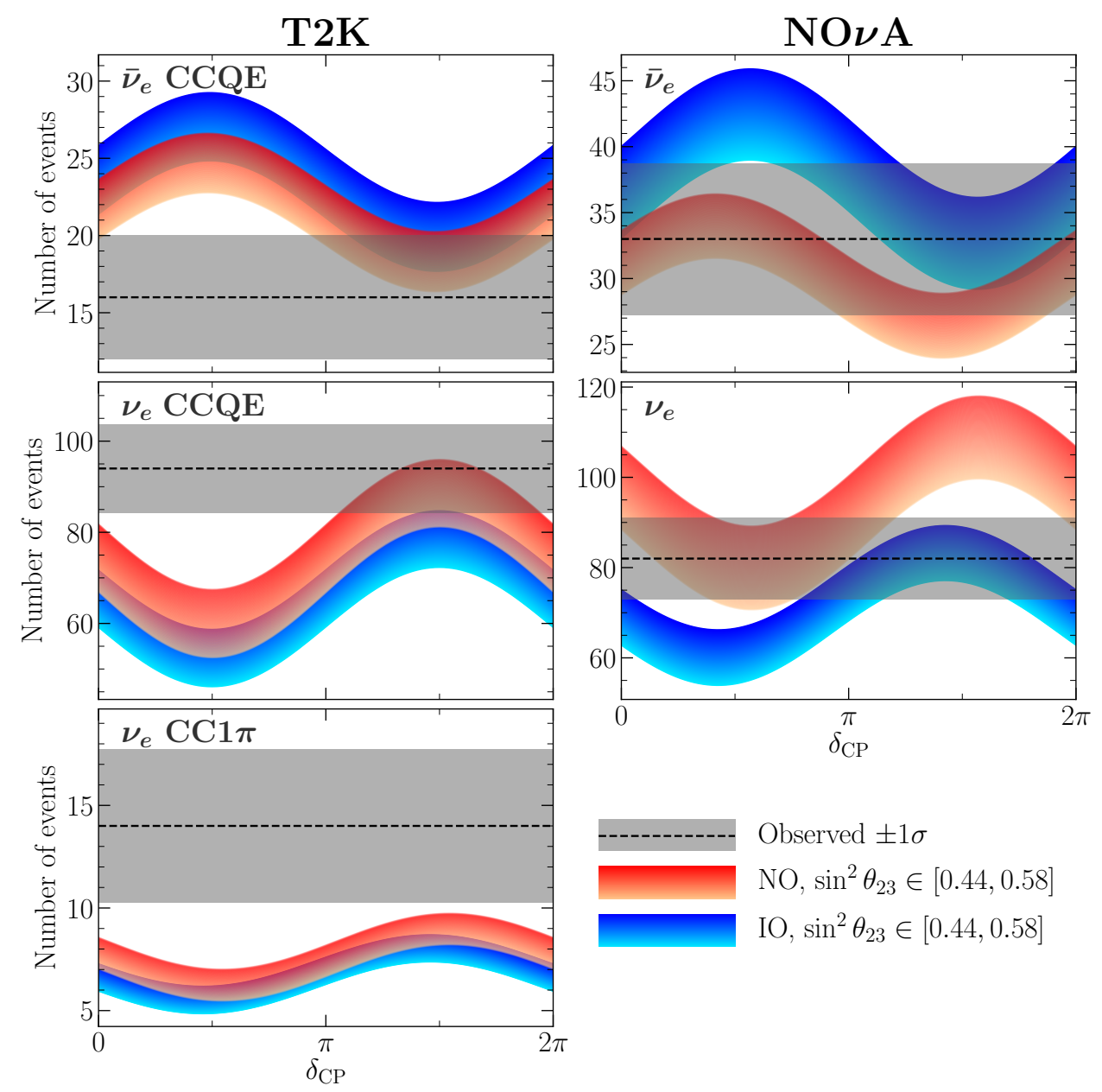

Figure 1. Predicted number of events as a function of $\delta_{\mathrm{CP}}$ for the T2K (left) and NOvA (right) appearance data sets. $\sin ^{2} \theta_{23}$ varies between 0.44 and 0.58 , where the lower-light (upper-dark) bound of the colored bands corresponds to 0.44 (0.58). Red (blue) bands correspond to NO (IO). For the other oscillation parameters we have adopted $\sin ^{2} \theta_{13}=0.0224,\left|\Delta m_{3 \ell}^{2}\right|=2.5 \times 10^{-3} \mathrm{eV}^{2}$, $\sin ^{2} \theta_{12}=0.310, \Delta m_{21}^{2}=7.39 \times 10^{-5} \mathrm{eV}^{2}$. The horizontal dashed lines show the observed number of events, with the $\pm 1 \sigma$ statistical error indicated by the gray shaded band.

NuFIT 4.1 shows a large excess of events in this sample, which has come down to $r=2.4$ with the latest data. Figure 1 still shows, that even the most favorable parameter choice cannot accomodate the observed number of events within $1 \sigma$. It seems that part of previous hints can be attributed to a statistical fluctuation in this sub-leading event sample. Let us stress, however, that due to the small $\mathrm{CC} 1 \pi$ event numbers, statistical uncertainties are large. Indeed, CCQE neutrino and anti-neutrino events consistently point in the same direction and they are both fitted best with $\mathrm{NO}$ and maximal $\mathrm{CP}$ phase.

Moving now to NOvA, we first observe from figure 1 the larger separation between the NO and IO bands compared to T2K. This is a manifestation of the increased matter effect because of the longer baseline in NOvA. Next, neutrino data have $r \approx 1$ which can be accommodated by $\left(\mathrm{NO}, \delta_{\mathrm{CP}} \simeq \pi / 2\right)$ or $\left(\mathrm{IO}, \delta_{\mathrm{CP}} \simeq 3 \pi / 2\right)$. This behavior is consistent with 

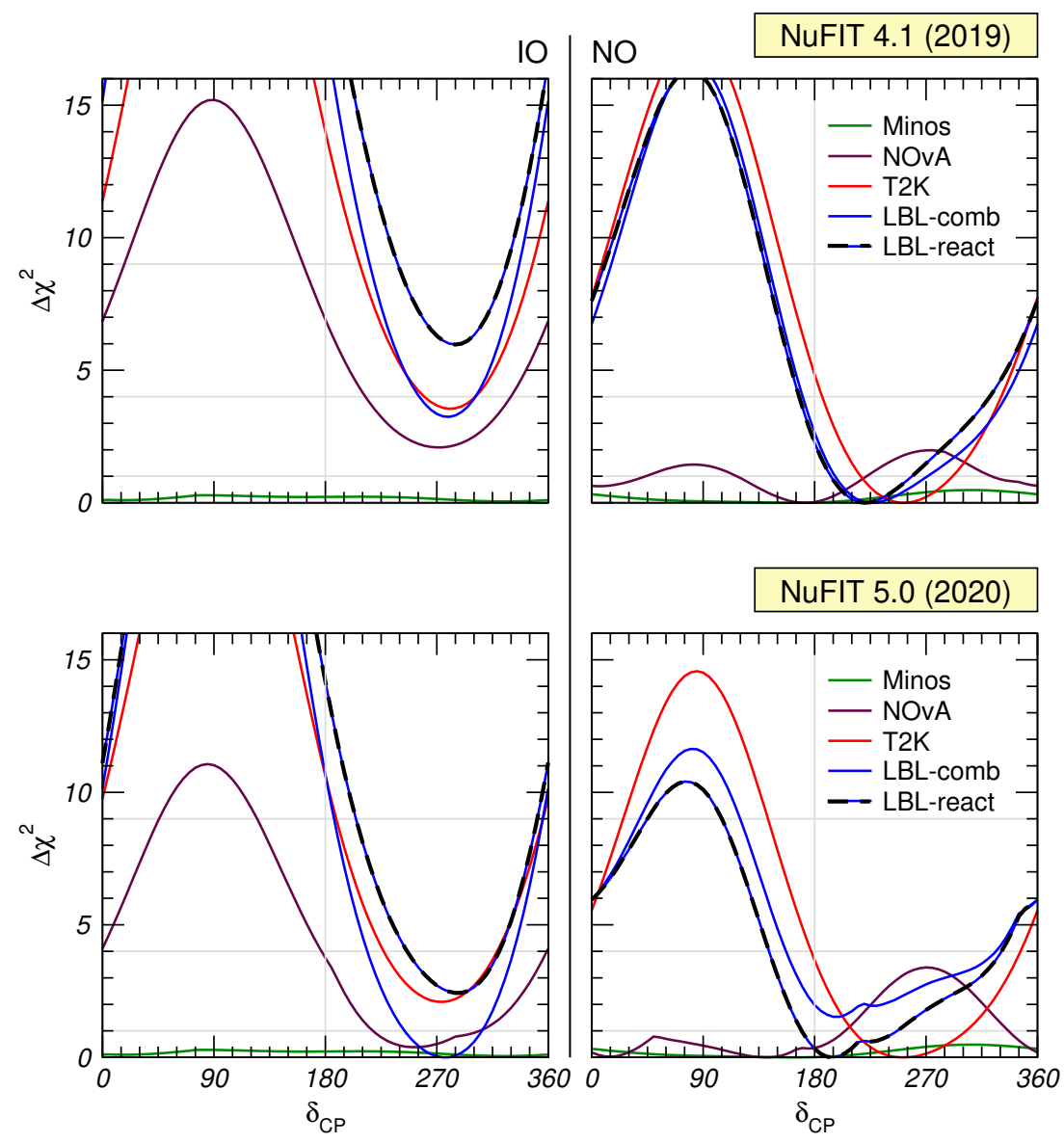

Figure 2. $\Delta \chi^{2}$ profiles as a function of $\delta_{\mathrm{CP}}$ for different LBL data sets and their combination. We have fixed $\sin ^{2} \theta_{13}=0.0224$ as well as the solar parameters and minimized with respect to $\theta_{23}$ and $\left|\Delta m_{3 \ell}^{2}\right|$. The black/blue dashed curves correspond to the combination of LBL data with the reactor experiments Day-aBay, RENO, Double-Chooz, and in this case also $\theta_{13}$ is left free in the fit. Left (right) panels are for IO (NO) and $\Delta \chi^{2}$ is shown with respect to the global best fit point for each curve. Upper panels are for the NuFIT 4.1 data set, whereas lower panels correspond to the current update.

NOvA anti-neutrinos, however in tension with $\mathrm{T} 2 \mathrm{~K}$ in the case of NO. We conclude from these considerations that the T2K and NOvA combination can be best fitted by IO and $\delta_{\mathrm{CP}} \simeq 3 \pi / 2$. This is indeed confirmed in figure 2 , showing the $\Delta \chi^{2}$ profiles as a function of $\delta_{\mathrm{CP}}$. We observe in the lower-right panel that NOvA disfavors $\left(\mathrm{NO}, \delta_{\mathrm{CP}} \simeq 3 \pi / 2\right)$ by about 4 units in $\chi^{2}$, whereas in the lower-left panel we see for IO consistent preference of T2K and NOvA for $\delta_{\mathrm{CP}} \simeq 3 \pi / 2$. For the combination this leads to a preferred best fit for IO with $\Delta \chi^{2}(\mathrm{NO}) \approx 1.5$ (which of course is not significant). We can also see that this effect was less relevant in NuFIT 4.1 (figure 2, upper panels) for which we had $r=1.3$ - compared to current 1.14 - for NOvA neutrino data. This slightly higher ratio allowed some more enhancement of the square-bracket in eq. (2.4) compared to the present situation, leading to less tension between $\mathrm{T} 2 \mathrm{~K}$ and NOvA for NO. It also lead to a larger significance of NOvA for NO. 



Figure 3. $1 \sigma$ and $2 \sigma$ allowed regions (2 dof) for T2K (red shading), NOvA (blue shading) and their combination (black curves). Contours are defined with respect to the local minimum for IO (left) or NO (right). We are fixing $\sin ^{2} \theta_{13}=0.0224, \sin ^{2} \theta_{12}=0.310, \Delta m_{21}^{2}=7.40 \times 10^{-5} \mathrm{eV}^{2}$ and minimize with respect to $\left|\Delta m_{3 \ell}^{2}\right|$.

The two-dimensional regions for T2K and NOvA in the $\left(\delta_{\mathrm{CP}}, \sin ^{2} \theta_{23}\right)$ plane for fixed $\theta_{13}$ are shown in figure 3. The better consistency for IO is apparent, while we stress that even for NO the $1 \sigma$ regions touch each other, indicating that also in this case the two experiments are statistically consistent. We are going to quantify this later in section 2.3.

\subsection{Accelerator versus reactor}

In the previous section we have discussed the status of the hints on $\mathrm{CP}$ violation and neutrino mass ordering in the latest LBL data. In the context of $3 \nu$ mixing the relevant oscillation probabilities for the LBL accelerator experiments depend also on $\theta_{13}$ which is most precisely determined from reactor experiments (and on the $\theta_{12}$ and $\Delta m_{21}^{2}$ parameters which are independently well constrained by solar and KamLAND data). So in our discussion, and also to construct the $\chi^{2}$ curves and regions shown in figures 2,3 , and 4 for T2K, NOvA, Minos, and the LBL-combination, those parameters are fixed to their current best fit values. Given the present precision in the determination of $\theta_{13}$ this yields very similar results to marginalize with respect to $\theta_{13}$, taking into account the information from reactor data by adding a Gaussian penalty term to the corresponding $\chi_{\mathrm{LBL}}^{2}$.

Let us stress that such procedure is not the same as making a combined analysis of LBL and reactor data, compare for instance the blue solid versus black/blue dashed curves in figure 2. This is so because relevant additional information on the mass ordering can be obtained from the comparison of $\nu_{\mu}$ and $\nu_{e}$ disappearance spectral data $[22,23]$. In brief, the relevant disappearance probabilities are approximately symmetric with respect to the sign of two effective mass-squared differences, usually denoted as $\Delta m_{\mu \mu}^{2}$ and $\Delta m_{e e}^{2}$, respectively. They are two different linear combinations of $\Delta m_{31}^{2}$ an $\Delta m_{32}^{2}$. Consequently, the precise determination of the oscillation frequencies in $\nu_{\mu}$ and $\nu_{e}$ disappearance experiments, yields 

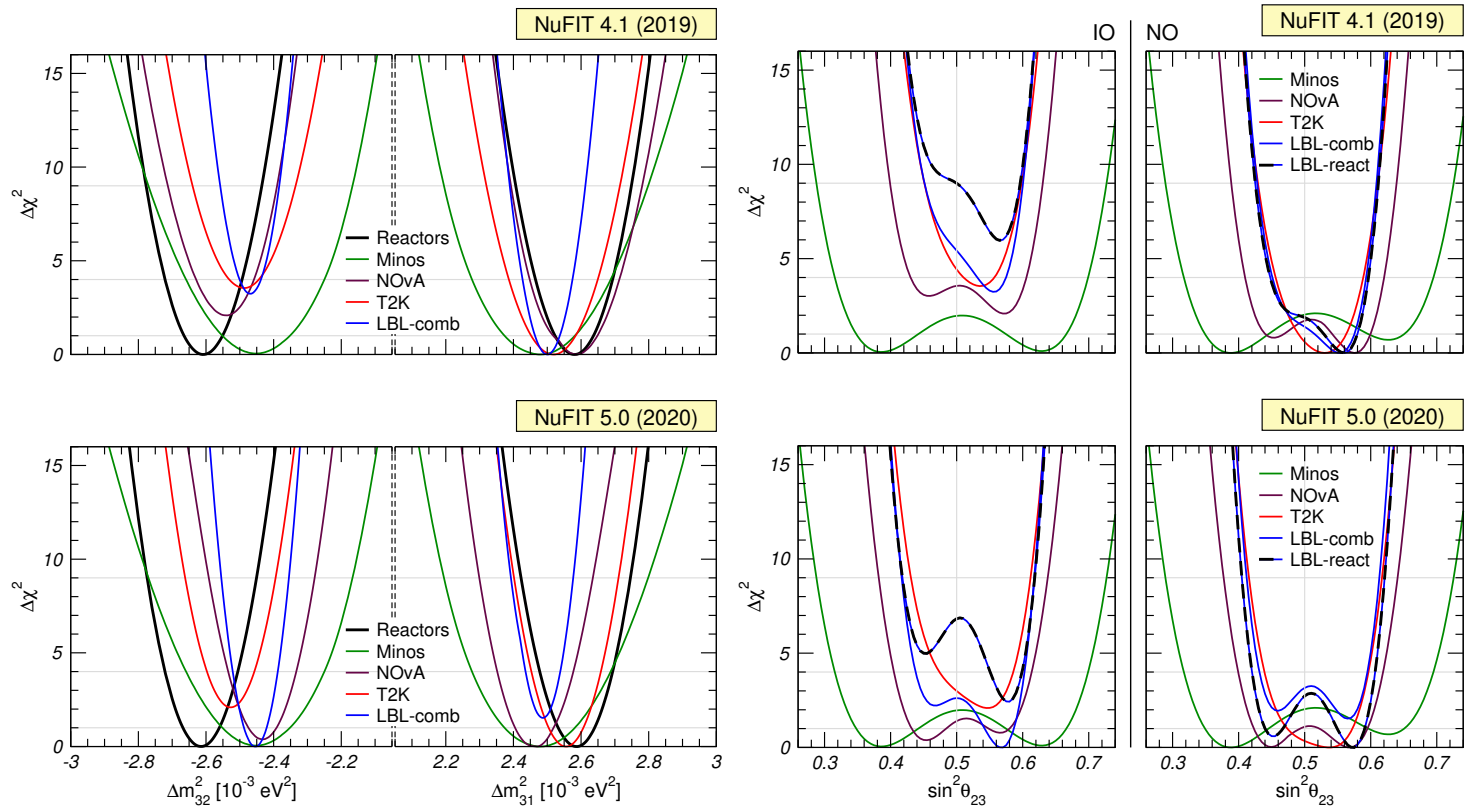

Figure 4. $\Delta \chi^{2}$ profiles as a function of $\Delta m_{3 \ell}^{2}$ (left) and $\sin ^{2} \theta_{23}$ (right) for different LBL data sets and their combination. In the left 4 panels we show also the combined reactor data from Daya-Bay, RENO and Double-Chooz. For all curves we have fixed $\sin ^{2} \theta_{13}=0.0224$ as well as the solar parameters and minimized with respect to the other un-displayed parameters. $\Delta \chi^{2}$ is shown with respect to the best fit mass ordering for each curve. Upper panels are for the NuFIT 4.1 data set, whereas lower panels correspond to the current update.

information on the sign of $\Delta m_{3 \ell}^{2}$. This effect has been present already in previous data (see, e.g., ref. [2] for a discussion). We see from the two lower-left panels of figure 4 that the region for $\left|\Delta m_{3 \ell}^{2}\right|$ for IO from the LBL combination (blue curve) is somewhat in tension with the one from the reactor experiments Daya-Bay, RENO and Double-Chooz (black curve), while they are in quite good agreement for NO.

In the accelerator-reactor combination this leads again to a best fit point for $\mathrm{NO}$, with $\Delta \chi^{2}(\mathrm{IO})=2.7$, considerably less than the value 6.2 of NuFIT 4.1 . This is explicitly shown, for example, in the LBL-reactor curves in figure 2. For the NO best fit, a compromise between T2K and NOvA appearance data has to be adopted, avoiding over-shoting the number of neutrino events in NOvA while still being able to accommodate both neutrino and anti-neutrino data from T2K, see figure 1. This leads to a shift of the allowed region towards $\delta_{\mathrm{CP}}=\pi$ and a rather wide allowed range for $\delta_{\mathrm{CP}}$ for NO, see figures 2 and 3 . On the other hand, we see from these figures that for IO, both T2K and NOvA prefer $\delta_{\mathrm{CP}} \simeq 270^{\circ}$. Consequently, if we restrict to this ordering, $\mathrm{CP}$ conservation remains disfavored at $\sim 3 \sigma$.

The behaviour as a function of $\sin ^{2} \theta_{23}$ is shown in figure 3 and the right panels of figure 4. It is mostly driven by the two T2K neutrino samples. As follows from eq. (2.4), their predicted event rate can be enhanced by increasing $\sin ^{2} \theta_{23}$. Therefore, in order to compensate for the reduction in IO, a slight preference for the second $\theta_{23}$ octant emerges for IO. In case of NO, this is less preferrable, since large $\sin ^{2} \theta_{23}$ would worsen the T2K anti-neutrino fit as well as NOvA neutrino data. 


\begin{tabular}{|l|ccc|ccc|}
\hline data sets & \multicolumn{3}{|c|}{ normal ordering } & \multicolumn{3}{c|}{ inverted ordering } \\
& $\chi_{\mathrm{PG}}^{2} / n$ & $p$-value & $\# \sigma$ & $\chi_{\mathrm{PG}}^{2} / n$ & $p$-value & $\# \sigma$ \\
\hline T2K vs NOvA & $6.7 / 4$ & 0.15 & $1.4 \sigma$ & $3.6 / 4$ & 0.46 & $0.7 \sigma$ \\
T2K vs React & $0.3 / 2$ & 0.87 & $0.2 \sigma$ & $2.5 / 2$ & 0.29 & $1.1 \sigma$ \\
NOvA vs React & $3.0 / 2$ & 0.23 & $1.2 \sigma$ & $6.2 / 2$ & 0.045 & $2.0 \sigma$ \\
T2K vs NOvA vs React & $8.4 / 6$ & 0.21 & $1.3 \sigma$ & $8.9 / 6$ & 0.18 & $1.3 \sigma$ \\
\hline T2K vs NOvA & $6.5 / 3$ & 0.088 & $1.7 \sigma$ & $2.8 / 3$ & 0.42 & $0.8 \sigma$ \\
T2K vs NOvA vs React & $7.8 / 4$ & 0.098 & $1.7 \sigma$ & $7.2 / 4$ & 0.13 & $1.5 \sigma$ \\
\hline
\end{tabular}

Table 2. Testing the consistency of different data sets shown in the first column assuming either normal or inverted ordering. "React" includes Daya-Bay, RENO and Double-Chooz. In the analyses above the horizontal line, $\theta_{13}$ is a free parameter, whereas below the line we have fixed $\sin ^{2} \theta_{13}=$ 0.0224. See text for more details.

\subsection{Consistency between T2K, NOvA and reactors}

Let us now address the question of whether some data sets are in tension with each other at a worrisome level. A useful method to quantify the consistency of different data sets is the so-called parameter goodness-of-fit (PG) [24]. It makes use of the following test statistic:

$$
\chi_{\mathrm{PG}}^{2}=\chi_{\min , \mathrm{glob}}^{2}-\sum_{i} \chi_{\min , i}^{2}
$$

where $i$ labels different data sets, $\chi_{\min , i}^{2}$ is the $\chi^{2}$ minimum of each data set individually, and $\chi_{\text {min,glob }}^{2}$ is the $\chi^{2}$ minimum of the global data, i.e., $\chi_{\text {min,glob }}^{2}=\min \left[\sum_{i} \chi_{i}^{2}\right]$. Let us denote by $n_{i}$ the number of model parameters on which the data set $i$ depends, and $n_{\text {glob }}$ the number of parameters on which the global data depends. Then the test statistic $\chi_{\mathrm{PG}}^{2}$ follows a $\chi^{2}$ distribution with $n$ degrees of freedom, where [24]

$$
n=\sum_{i} n_{i}-n_{\text {glob }}
$$

We are going to apply this test now to different combination of the three data sets, "T2K", "NOvA", and "React", where "React" is the joint data set of Daya-Bay, RENO and Double-Chooz. ${ }^{3}$ The accelerator samples always include appearance and disappearance channels for both neutrinos and anti-neutrinos. In order to study the consistency of the sets under a given hypothesis for the neutrino mass ordering, all minimizations are preformed restricting to a given mass ordering. Furthermore, the solar parameters are kept fixed and hence, we have $n_{\mathrm{T} 2 \mathrm{~K}}=n_{\mathrm{NOvA}}=n_{\text {glob }}=4$ (namely $\left.\theta_{13}, \theta_{23}, \delta_{\mathrm{CP}},\left|\Delta m_{3 \ell}^{2}\right|\right)$ and $n_{\text {React }}=2$ (namely $\theta_{13},\left|\Delta m_{3 \ell}^{2}\right|$ ). The results are shown in table 2 .

First, we check the pair-wise consistency of two out of the three sets. In all cases we find perfect consistency with $p$-values well above $10 \%$. The only exception is NOvA vs React for IO which show tension at the $2 \sigma$ level. A large contribution to this effect comes

\footnotetext{
${ }^{3}$ We have also checked that the three reactor experiments by themselves are in excellent agreement with each other, see the figure "Synergies: atmospheric mass-squared splitting" available at [11]. This justifies to merge them into a single set.
} 
from the determination of $\Delta m_{3 \ell}^{2}$, which agrees better for NO than for IO, see figure 4 (lower-left panels). The consistency of all three sets (T2K vs NOvA vs React) is excellent for both orderings.

Second, we perform an analysis for fixed $\sin ^{2} \theta_{13}=0.0224$ for all data sets. Since the accelerator experiments provide a comparatively weak constraint on $\theta_{13}$ we want to remove this freedom from the T2K and NOvA fits and test the consistency under the hypothesis of fixed $\theta_{13}$. Under this assumption, all $n_{i}$ as well as $n_{\text {glob }}$ quoted above are reduced by 1 . The results of this analysis are shown in the lower part of table 2. Testing T2K vs NOvA under this assumption, we find better compatibility for IO, consistent with the discussion above and figures 2 and 3. Let us stress, however, that even for NO the $p$-value is $9 \%$, indicating consistency at the $1.7 \sigma$ level. Hence, we find no severe tension between T2K and NOvA. Finally, the joint T2K vs NOvA vs React analysis with fixed $\theta_{13}$ reveals roughly equal good consistency among the three sets for both orderings, at around $1.5 \sigma$. For NO the very slight tension is driven by $\mathrm{T} 2 \mathrm{~K}$ vs NOvA, whereas for IO the reactor/accelerator complementarity in the determination of $\Delta m_{3 \ell}^{2}$ provides a few units to $\chi_{\mathrm{PG}}^{2}$.

To conclude this discussion, we find that all involved data sets are perfectly statistically compatible under the hypothesis of three-flavor oscillations.

\section{Resolved tension in the solar sector}

The analyses of the solar experiments and of KamLAND give the dominant contribution to the determination of $\Delta m_{21}^{2}$ and $\theta_{12}$. It has been a result of global analyses for the last decade, that the value of $\Delta m_{21}^{2}$ preferred by KamLAND was somewhat higher than the one from solar experiments. The tension appeared due to a combination of two effects: the well-known fact that the ${ }^{8} \mathrm{~B}$ measurements performed by SNO, SK and Borexino showed no evidence of the low energy spectrum turn-up expected in the standard LMA-MSW [21, 25] solution for the value of $\Delta m_{21}^{2}$ favored by KamLAND, and the observation of a nonvanishing day-night asymmetry in SK, whose size is larger than the one predicted for the $\Delta m_{21}^{2}$ value indicated by KamLAND. In our last published analysis [2] we included the energy-zenith spectra or day/night spectra for SK1-3, together with the 2860-day total energy spectrum of SK4 [26]. This last one made the lack of the turn-up effect slightly stronger. As for the day-night variation in SK4, it was included in terms of their quoted day-night asymmetry for SK4 2055-day [27]

$$
A_{\mathrm{D} / \mathrm{N}, \mathrm{SK} 4-2055}=[-3.1 \pm 1.6(\text { stat. }) \pm 1.4(\text { syst. })] \% \text {. }
$$

Altogether this resulted in slightly over $2 \sigma$ discrepancy between the best fit $\Delta m_{21}^{2}$ value indicated of KamLAND and the solar results. For example the best fit $\Delta m_{21}^{2}$ of KamLAND was at $\Delta \chi_{\text {solar }}^{2}=4.7$ in the analysis with the GS98 fluxes.

Here we update the solar analysis to include the latest SK4 2970-day results ${ }^{4}$ presented in Neutrino2020 [16] in the form of their total energy spectrum and the updated day-night

\footnotetext{
${ }^{4}$ We do not include here the latest data release from Borexino [28], which is expected to have a very small impact on the determination of oscillation parameters.
} 

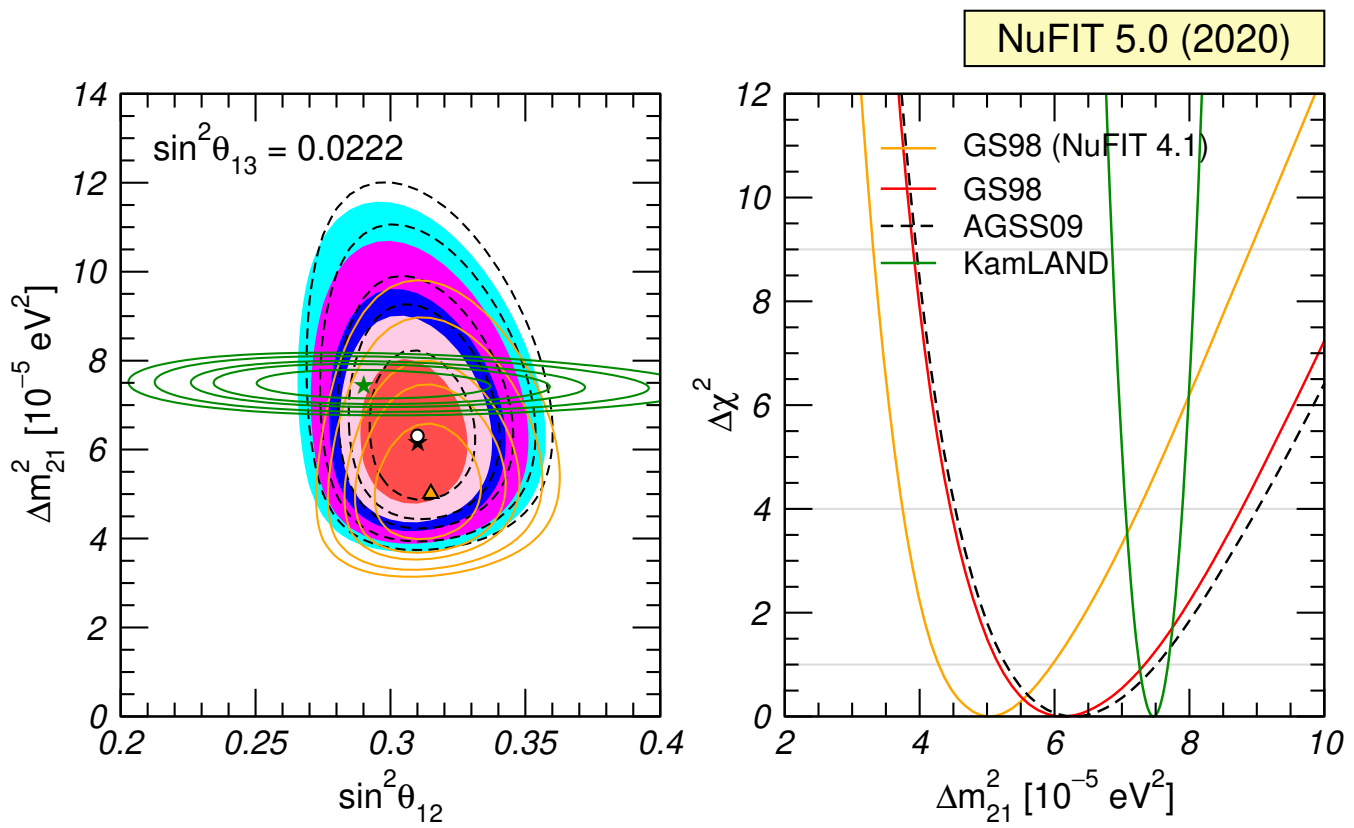

Figure 5. Left: allowed parameter regions (at $1 \sigma, 90 \%, 2 \sigma, 99 \%$, and $3 \sigma$ CL for 2 dof) from the combined analysis of solar data for GS98 model (full regions with best fit marked by black star) and AGSS09 model (dashed void contours with best fit marked by a white dot), and for the analysis of KamLAND data (solid green contours with best fit marked by a green star) for fixed $\sin ^{2} \theta_{13}=0.0224\left(\theta_{13}=8.6\right)$. We also show as orange contours the previous results of the global analysis for the GS98 model in ref. . [2]. Right: $\Delta \chi^{2}$ dependence on $\Delta m_{21}^{2}$ for the same four analyses after marginalizing over $\theta_{12}$.

asymmetry

$$
A_{\mathrm{D} / \mathrm{N}, \mathrm{SK} 4-2970}=(-2.1 \pm 1.1) \% \text {. }
$$

We show in figure 5 the present determination of these parameters from the global solar analysis in comparison with that of KamLAND data. The results of the solar neutrino analysis are shown for the two latest versions of the Standard Solar Model, namely the GS98 and the AGSS09 models [29] obtained with two different determinations of the solar abundances [30]. For sake of comparison we also show the corresponding results of the solar analysis with the pre-Neutrino2020 data [2].

As seen in the figure, with the new data the tension between the best fit $\Delta m_{21}^{2}$ of KamLAND and that of the solar results has decreased. Quantitatively we now find that the best fit $\Delta m_{21}^{2}$ of KamLAND lies at $\Delta \chi_{\text {solar }}^{2}=1.3(1.14 \sigma)$ in the analysis with the GS98 fluxes. This decrease in the tension is due to both, the smaller day-night asymmetry (which lowers $\Delta \chi_{\text {solar }}^{2}$ of the best fit $\Delta m_{21}^{2}$ of KamLAND by 2.4 units) and the slightly more pronounced turn-up in the low energy part of the spectrum which lowers it one extra unit.

\section{Global fit results}

Finally we present a selection of the results of our global analysis NuFIT 5.0 using data available up to July 2020 (see appendix A for the complete list of the used data including 
references). We show two versions of the analysis which differ in the inclusion of the results of the Super-Kamiokande atmospheric neutrino data (SK-atm). As discussed in ref. [2] there is not enough information available for us to make an independent analysis comparable in detail to that performed by the collaboration, hence we have been making use of their tabulated $\chi^{2}$ map which we can combine with our global analysis for the rest of experiments. This table was made available for their analysis of SK1-4 corresponding to 328 kton-years data [31]. The collaboration has presented new oscillation results obtained from the analysis of updated SK4 samples, both by itself [32] and in combination with the SK1-3 phases [16]. They seem to indicate that their hint for ordering discrimination has also decreased. Unfortunately the corresponding $\chi^{2}$ maps of these analyses have not been made public. Hence in what follows we refer as "with SK-atm" to the analysis including the tabulated SK1-4 328 kiloton years data $\chi^{2}$ map, i.e., the same as in NuFIT 4.0 and 4.1.

Here we graphically present the results of our global analysis in the form of onedimensional $\Delta \chi^{2}$ curves (figure 6) and two-dimensional projections of confidence regions (figure 7). The corresponding best fit values as well as $1 \sigma$ and $3 \sigma$ confidence intervals for the oscillation parameters are listed in table $3{ }^{5}$ Defining the $3 \sigma$ relative precision of the parameter by $2\left(x^{\text {up }}-x^{\text {low }}\right) /\left(x^{\text {up }}+x^{\text {low }}\right)$, where $x^{\text {up }}\left(x^{\text {low }}\right)$ is the upper (lower) bound on a parameter $x$ at the $3 \sigma$ level, we obtain the following $3 \sigma$ relative precision (marginalizing over ordering):

$$
\begin{aligned}
\theta_{12}: 14 \%, & \theta_{13}: 9.0 \%, & \theta_{23}: 27 \%[25 \%], \\
\Delta m_{21}^{2}: 16 \%, & \left|\Delta m_{3 \ell}^{2}\right|: 6.7 \%[6.5 \%], & \delta_{\mathrm{CP}}: 100 \%[100 \%],
\end{aligned}
$$

where the numbers between brackets show the impact of including SK-atm in the precision of the determination of such parameter. The $\Delta \chi^{2}$ profile of $\delta_{\mathrm{CP}}$ is not gaussian and hence its precision estimation above is only indicative.

In table 3 we give the best fit values and confidence intervals for both mass orderings, relative to the local best fit points in each ordering. The global confidence intervals (marginalizing also over the ordering) are identical to the ones for normal ordering, which have also been used in eq. (4.1). The only exception to this statement is $\Delta m_{3 \ell}^{2}$ in the analysis without SK-atm: in this case a disconnected interval would appear above $2 \sigma$ corresponding to negative values of $\Delta m_{3 \ell}^{2}$ (i.e., inverted ordering).

Projecting over the combinations appearing on the elements of the leptonic mixing matrix we derive the following $3 \sigma$ ranges (see ref. [33] for details on how we derive the ranges) on their magnitude:

$$
\begin{aligned}
& |U|_{3 \sigma}^{\mathrm{w} / \mathrm{o} \text { SK-atm }}=\left(\begin{array}{lll}
0.801 \rightarrow 0.845 & 0.513 \rightarrow 0.579 & 0.143 \rightarrow 0.156 \\
0.233 \rightarrow 0.507 & 0.461 \rightarrow 0.694 & 0.631 \rightarrow 0.778 \\
0.261 \rightarrow 0.526 & 0.471 \rightarrow 0.701 & 0.611 \rightarrow 0.761
\end{array}\right) \\
& |U|_{3 \sigma}^{\text {with SK-atm }}=\left(\begin{array}{lll}
0.801 \rightarrow 0.845 & 0.513 \rightarrow 0.579 & 0.143 \rightarrow 0.155 \\
0.234 \rightarrow 0.500 & 0.471 \rightarrow 0.689 & 0.637 \rightarrow 0.776 \\
0.271 \rightarrow 0.525 & 0.477 \rightarrow 0.694 & 0.613 \rightarrow 0.756
\end{array}\right)
\end{aligned}
$$

\footnotetext{
${ }^{5}$ For additional figures and tables corresponding to this global analysis we refer the reader to the NuFIT webpage [11].
} 

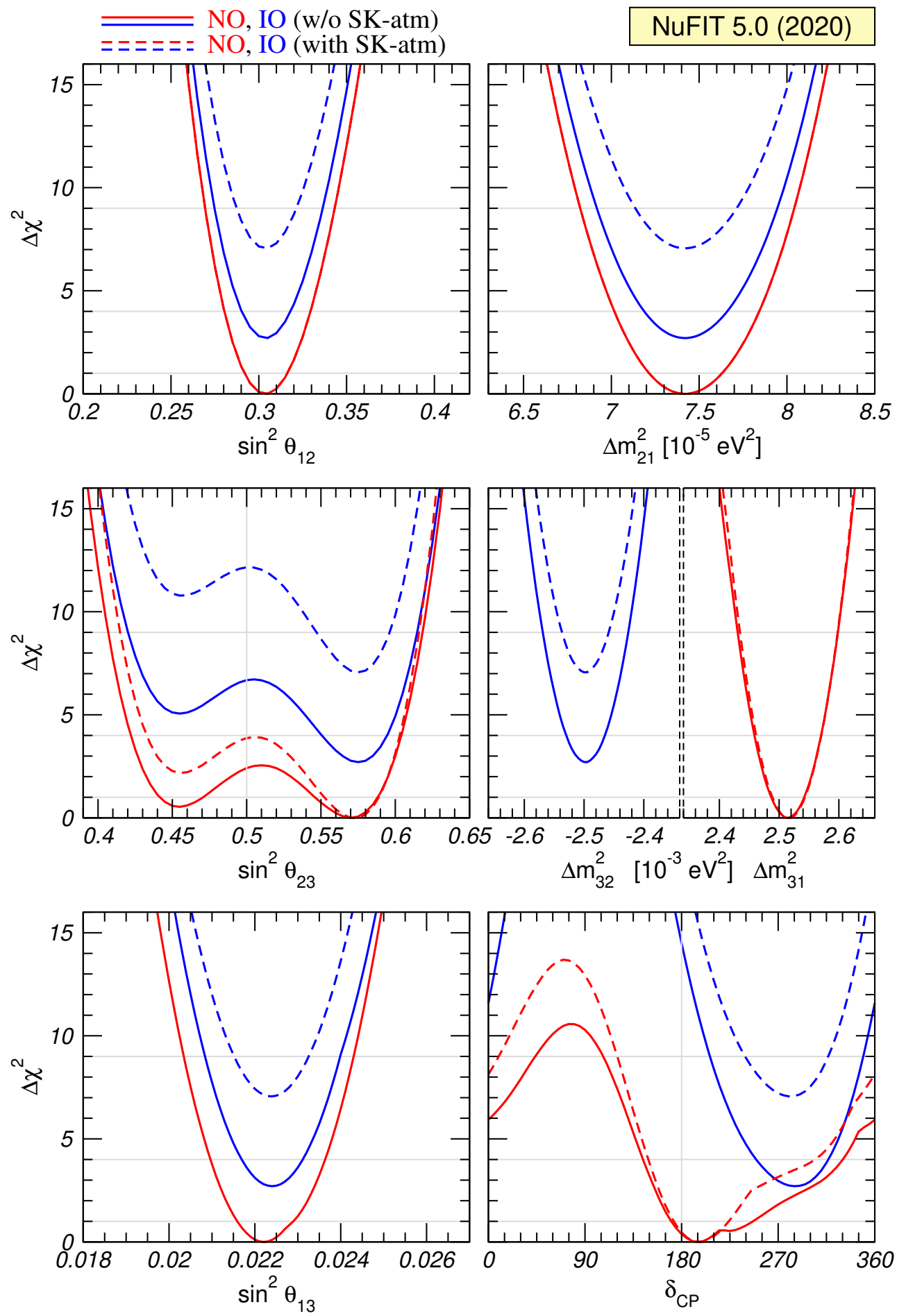

Figure 6. Global $3 \nu$ oscillation analysis. We show $\Delta \chi^{2}$ profiles minimized with respect to all undisplayed parameters. The red (blue) curves correspond to Normal (Inverted) Ordering. Solid (dashed) curves are without (with) adding the tabulated SK-atm $\Delta \chi^{2}$. Note that as atmospheric mass-squared splitting we use $\Delta m_{31}^{2}$ for NO and $\Delta m_{32}^{2}$ for IO. 


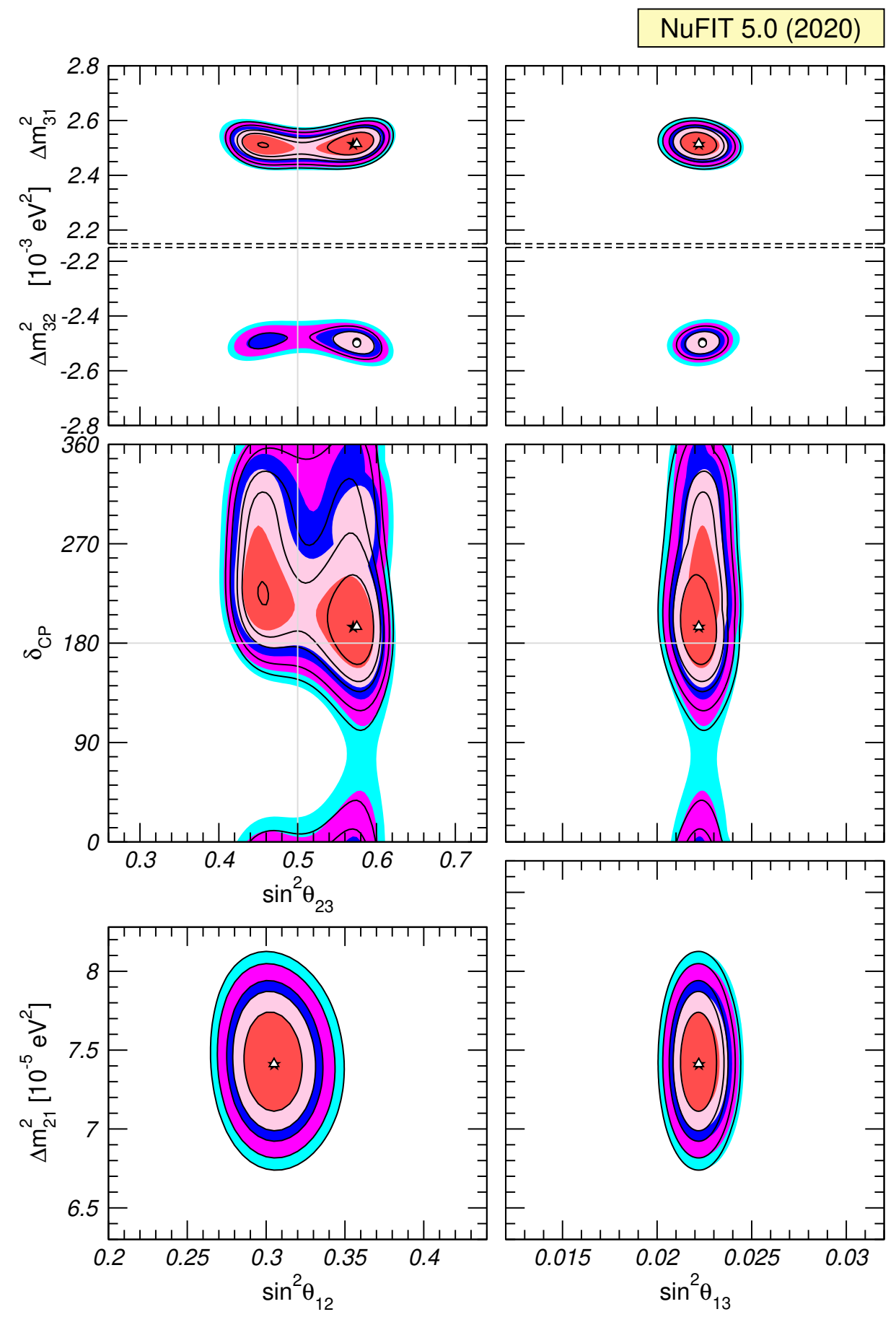

Figure 7. Global $3 \nu$ oscillation analysis. Each panel shows the two-dimensional projection of the allowed six-dimensional region after minimization with respect to the undisplayed parameters. The regions in the four lower panels are obtained from $\Delta \chi^{2}$ minimized with respect to the mass ordering. The different contours correspond to $1 \sigma, 90 \%, 2 \sigma, 99 \%, 3 \sigma$ CL (2 dof). Colored regions (black contour curves) are without (with) adding the tabulated SK-atm $\Delta \chi^{2}$. Note that as atmospheric mass-squared splitting we use $\Delta m_{31}^{2}$ for NO and $\Delta m_{32}^{2}$ for IO. 


\begin{tabular}{|c|c|c|c|c|c|}
\hline \multirow{11}{*}{ 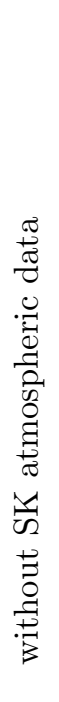 } & & \multicolumn{2}{|c|}{ Normal Ordering (best fit) } & \multicolumn{2}{|c|}{ Inverted Ordering $\left(\Delta \chi^{2}=2.7\right)$} \\
\hline & & $\mathrm{bfp} \pm 1 \sigma$ & $3 \sigma$ range & bfp $\pm 1 \sigma$ & $3 \sigma$ range \\
\hline & $\sin ^{2} \theta_{12}$ & $0.304_{-0.012}^{+0.013}$ & $0.269 \rightarrow 0.343$ & $0.304_{-0.012}^{+0.013}$ & $0.269 \rightarrow 0.343$ \\
\hline & $\theta_{12} /^{\circ}$ & $33.44_{-0.75}^{+0.78}$ & $31.27 \rightarrow 35.86$ & $33.45_{-0.75}^{+0.78}$ & $31.27 \rightarrow 35.87$ \\
\hline & $\sin ^{2} \theta_{23}$ & $0.570_{-0.024}^{+0.018}$ & $0.407 \rightarrow 0.618$ & $0.575_{-0.021}^{+0.017}$ & $0.411 \rightarrow 0.621$ \\
\hline & $\theta_{23} /^{\circ}$ & $49.0_{-1.4}^{+1.1}$ & $39.6 \rightarrow 51.8$ & $49.3_{-1.2}^{+1.0}$ & $39.9 \rightarrow 52.0$ \\
\hline & $\sin ^{2} \theta_{13}$ & $0.02221_{-0.00062}^{+0.00068}$ & $0.02034 \rightarrow 0.02430$ & $0.02240_{-0.00062}^{+0.00062}$ & $0.02053 \rightarrow 0.02436$ \\
\hline & $\theta_{13} /{ }^{\circ}$ & $8.57_{-0.12}^{+0.13}$ & $8.20 \rightarrow 8.97$ & $8.61_{-0.12}^{+0.12}$ & $8.24 \rightarrow 8.98$ \\
\hline & $\delta_{\mathrm{CP}} /^{\circ}$ & $195_{-25}^{+51}$ & $107 \rightarrow 403$ & $286_{-32}^{+27}$ & $192 \rightarrow 360$ \\
\hline & $\frac{\Delta m_{21}^{2}}{10^{-5} \mathrm{eV}^{2}}$ & $7.42_{-0.20}^{+0.21}$ & $6.82 \rightarrow 8.04$ & $7.42_{-0.20}^{+0.21}$ & $6.82 \rightarrow 8.04$ \\
\hline & $\frac{\Delta m_{3 \ell}^{2}}{10^{-3} \mathrm{eV}^{2}}$ & $+2.514_{-0.027}^{+0.028}$ & $+2.431 \rightarrow+2.598$ & $-2.497_{-0.028}^{+0.028}$ & $-2.583 \rightarrow-2.412$ \\
\hline \multirow{11}{*}{ 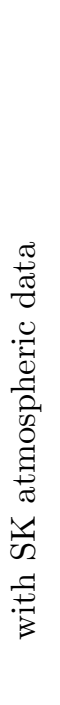 } & & \multicolumn{2}{|c|}{ Normal Ordering (best fit) } & \multicolumn{2}{|c|}{ Inverted Ordering $\left(\Delta \chi^{2}=7.1\right)$} \\
\hline & & bfp $\pm 1 \sigma$ & $3 \sigma$ range & bfp $\pm 1 \sigma$ & $3 \sigma$ range \\
\hline & $\sin ^{2} \theta_{12}$ & $0.304_{-0.012}^{+0.012}$ & $0.269 \rightarrow 0.343$ & $0.304_{-0.012}^{+0.013}$ & $0.269 \rightarrow 0.343$ \\
\hline & $\theta_{12} /^{\circ}$ & $33.44_{-0.74}^{+0.77}$ & $31.27 \rightarrow 35.86$ & $33.45_{-0.75}^{+0.78}$ & $31.27 \rightarrow 35.87$ \\
\hline & $\sin ^{2} \theta_{23}$ & $0.573_{-0.020}^{+0.016}$ & $0.415 \rightarrow 0.616$ & $0.575_{-0.019}^{+0.016}$ & $0.419 \rightarrow 0.617$ \\
\hline & $\theta_{23} /^{\circ}$ & $49.2_{-1.2}^{+0.9}$ & $40.1 \rightarrow 51.7$ & $49.3_{-1.1}^{+0.9}$ & $40.3 \rightarrow 51.8$ \\
\hline & $\sin ^{2} \theta_{13}$ & $0.02219_{-0.00063}^{+0.00062}$ & $0.02032 \rightarrow 0.02410$ & $0.02238_{-0.00062}^{+0.00063}$ & $0.02052 \rightarrow 0.02428$ \\
\hline & $\theta_{13} /^{\circ}$ & $8.57_{-0.12}^{+0.12}$ & $8.20 \rightarrow 8.93$ & $8.60_{-0.12}^{+0.12}$ & $8.24 \rightarrow 8.96$ \\
\hline & $\delta_{\mathrm{CP}} /{ }^{\circ}$ & $197_{-24}^{+27}$ & $120 \rightarrow 369$ & $282_{-30}^{+26}$ & $193 \rightarrow 352$ \\
\hline & $\frac{\Delta m_{21}^{2}}{10^{-5} \mathrm{eV}^{2}}$ & $7.42_{-0.20}^{+0.21}$ & $6.82 \rightarrow 8.04$ & $7.42_{-0.20}^{+0.21}$ & $6.82 \rightarrow 8.04$ \\
\hline & $\frac{\Delta m_{3 \ell}^{2}}{10^{-3} \mathrm{eV}^{2}}$ & $+2.517_{-0.028}^{+0.026}$ & $+2.435 \rightarrow+2.598$ & $-2.498_{-0.028}^{+0.028}$ & $-2.581 \rightarrow-2.414$ \\
\hline
\end{tabular}

Table 3. Three-flavor oscillation parameters from our fit to global data. The numbers in the 1st (2nd) column are obtained assuming NO (IO), i.e., relative to the respective local minimum. Note that $\Delta m_{3 \ell}^{2} \equiv \Delta m_{31}^{2}>0$ for NO and $\Delta m_{3 \ell}^{2} \equiv \Delta m_{32}^{2}<0$ for IO. The results shown in the upper (lower) table are without (with) adding the tabulated SK-atm $\Delta \chi^{2}$.

Note that there are strong correlations between these allowed ranges due to the unitary constraint.

The present status of leptonic CP violation is further illustrated in figure 8 where we show the determination of the Jarlskog invariant defined as:

$$
\begin{aligned}
J_{\mathrm{CP}} & \equiv \operatorname{Im}\left[U_{\alpha i} U_{\alpha j}^{*} U_{\beta i}^{*} U_{\beta j}\right] \\
& \equiv J_{\mathrm{CP}}^{\max } \sin \delta_{\mathrm{CP}}=\cos \theta_{12} \sin \theta_{12} \cos \theta_{23} \sin \theta_{23} \cos ^{2} \theta_{13} \sin \theta_{13} \sin \delta_{\mathrm{CP}} .
\end{aligned}
$$

It provides a convention-independent measure of leptonic $\mathrm{CP}$ violation in neutrino propagation in vacuum [34] — analogous to the factor introduced in ref. [35] for the description of $\mathrm{CP}$ violating effects in the quark sector, presently determined to be $J_{\mathrm{CP}}^{\text {quarks }}=$ 

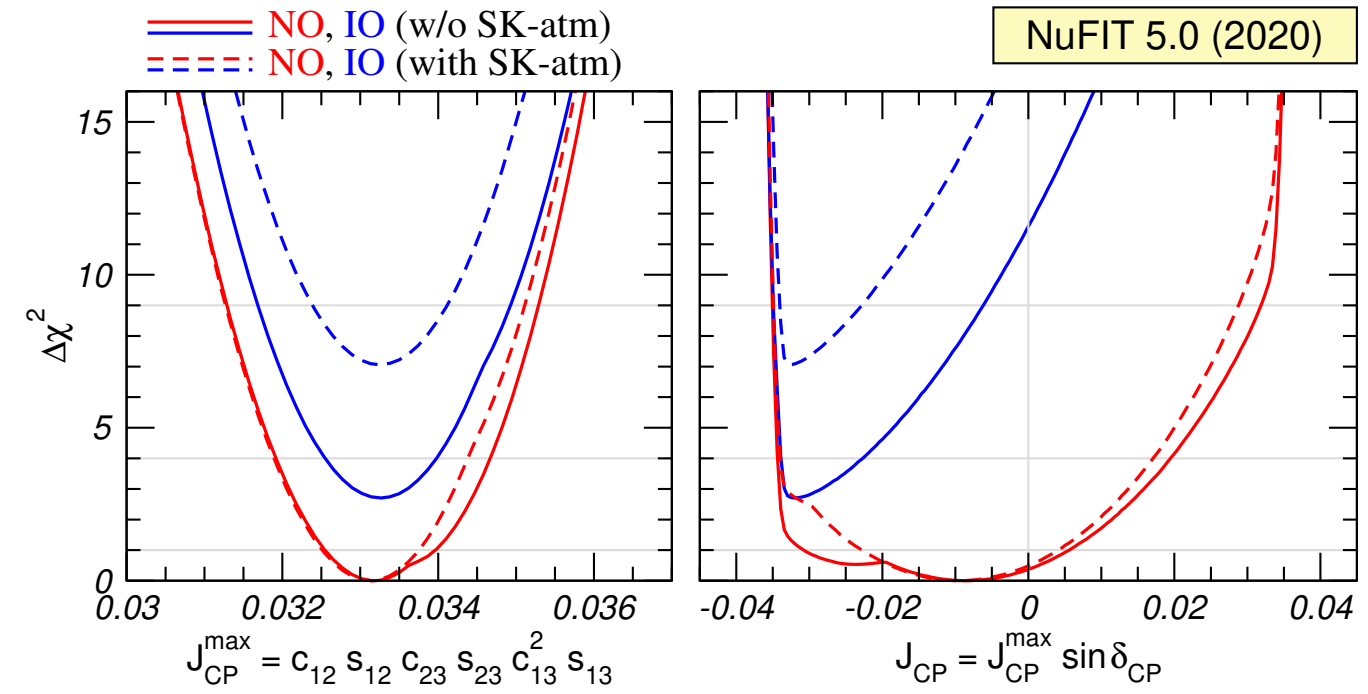

Figure 8. Dependence of the global $\Delta \chi^{2}$ function on the Jarlskog invariant. The red (blue) curves are for NO (IO). Solid (dashed) curves are without (with) adding the tabulated SK-atm $\Delta \chi^{2}$.

$(3.18 \pm 0.15) \times 10^{-5}[36]$. From the figure we read that the determination of the mixing angles implies a maximal possible value of the Jarlskog invariant of

$$
J_{\mathrm{CP}}^{\max }=0.0332 \pm 0.0008( \pm 0.0019)
$$

at $1 \sigma(3 \sigma)$ for both orderings. Furthermore we see that with the inclusion of the new results, the best fit value $J_{\mathrm{CP}}^{\text {best }}=-0.0089$ is only favored over CP conservation $J_{\mathrm{CP}}=0$ with $\Delta \chi^{2}=0.38$, irrespective of SK-atm.

\section{Summary}

Let us summarize the main findings resulting from the Neutrino2020 updates in neutrino oscillations.

- The best fit in the global analysis remains for the normal mass ordering, however, with reduced significance. In the global analysis without SK-atm, inverted ordering is disfavored only with a $\Delta \chi^{2}=2.7(1.6 \sigma)$ to be compared with $\Delta \chi^{2}=6.2(2.5 \sigma)$ in NuFIT 4.1. This change is driven by the new LBL results from T2K and NOvA which indeed by themselves favor IO (with $\theta_{13}$ as determined by the reactor data and $\theta_{12}$ and $\Delta m_{21}^{2}$ by the solar and KamLAND results). The best fit for NO in the combined global analysis is driven by the better compatibility between the $\Delta m_{3 \ell}^{2}$ determined in $\nu_{\mu}$ disappearance at accelerators with that from $\nu_{e}$ disappearance at reactors (see left panel in figure 4).

- Despite slightly different tendencies in some parameter regions, T2K, NOvA and reactor experiments are statistically in very good agreement with each other. We have performed tests of various experiment and analysis combinations, which all show consistency at a CL below $2 \sigma$ (section 2.3). 
- If atmospheric data from Super-Kamiokande is included, inverted ordering is disfavored with a $\Delta \chi^{2}=7.3(2.7 \sigma)$ compared to $\Delta \chi^{2}=10.4(3.2 \sigma)$ in NuFIT 4.1. Hence, a modest indication for NO remains. Let us note that in the recent Super-Kamiokande update presented at Neutrino2020 [16] (with increased statistic and improved mass ordering sensitivity) the $\Delta \chi^{2}$ for IO is reduced by about 1 unit compared to the analysis we are using in our global fit. Therefore we expect that once the $\chi^{2}$ map for the new SK analysis becomes available, the combined hint in favor of NO may further decrease.

- We obtain a very mild preference for the second octant of $\theta_{23}$, with the best fit point located at $\sin ^{2} \theta_{23}=0.57$ (slightly more non-maximal than the best fit of 0.56 in NuFIT 4.1), but with the local minimum in the first octant at $\sin ^{2} \theta_{23}=0.455$ at a $\Delta \chi^{2}=0.53(2.2)$ without (with) SK-atm. Maximal mixing $\left(\sin ^{2} \theta_{23}=0.5\right)$ is disfavored with $\Delta \chi^{2}=2.4$ (3.9) without (with) SK-atm.

- The best fit for the complex phase is at $\delta_{\mathrm{CP}}=195^{\circ}$. Compared to previous results (e.g., NuFIT 4.1 [11]), the allowed range is pushed towards the CP conserving value of $180^{\circ}$, which is now allowed at $0.6 \sigma$ with or without SK-atm. If we restrict to $\mathrm{IO}$, the best fit of $\delta_{\mathrm{CP}}$ remains close to maximal $\mathrm{CP}$ violation, with $\mathrm{CP}$ conservation being disfavored at around $3 \sigma$.

- New solar neutrino data from Super-Kamiokande lead to an upward shift of the allowed region for $\Delta m_{21}^{2}$, which significantly decreased the tension between solar and KamLAND data. They are now compatible at $1.1 \sigma$, compared to about $2.2 \sigma$ for the pre-Neutrino2020 situation.

Overall we have witnessed decreasing significance of various "hints" present in previous data. This is consistent with the fate of fluctuations which is that of fading away as time goes by.

\section{Acknowledgments}

We thank Anatael Cabrera and Jonghee Yoo for correspondence conerning the DoubleChooz and RENO analyses, respectively. AZ acknowledges helpful correspondence with Alvaro Hernandez-Cabezudo on the RENO fit. This work was supported by the spanish grants FPA2016-76005-C2-1-P, FPA2016-78645-P, and PID2019-105614GB-C21, by USANSF grant PHY-1915093, by AGAUR (Generalitat de Catalunya) grant 2017-SGR-929. IE acknowledges support from the FPU program fellowship FPU15/0369. AZ acknowledges support by the doctoral school KSETA from funds of the GSSP programme of DAAD. The authors acknowledge the support of the Spanish Agencia Estatal de Investigacion through the grant "IFT Centro de Excelencia Severo Ochoa SEV-2016-0597". 


\section{A List of data used in the analysis}

\section{Solar experiments}

- External information: standard Solar Model [30].

- Chlorine total rate [37], 1 data point.

- Gallex \& GNO total rates [38], 2 data points.

- SAGE total rate [39], 1 data point.

- SK1 full energy and zenith spectrum [40], 44 data points.

- SK2 full energy and day/night spectrum [41], 33 data points.

- SK3 full energy and day/night spectrum [42], 42 data points.

- SK4 2970-day day-night asymmetry [16] and energy spectrum [16], 24 data points.

- SNO combined analysis [43], 7 data points.

- Borexino Phase-I 741-day low-energy data [44], 33 data points.

- Borexino Phase-I 246-day high-energy data [45], 6 data points.

- Borexino Phase-II 408-day low-energy data [46], 42 data points.

\section{Atmospheric experiments}

- External information: atmospheric neutrino fluxes [47].

- IceCube/DeepCore 3-year data [48, 49], 64 data points.

- SK1-4 328 kiloton years [50], $\chi^{2}$ map [31] added to our global analysis.

\section{Reactor experiments}

- KamLAND separate DS1, DS2, DS3 spectra [51] with Daya-Bay reactor $\nu$ fluxes [52], 69 data points.

- Double-Chooz FD/ND spectral ratio, with 1276-day (FD), 587-day (ND) exposures [13], 26 data points.

- Daya-Bay 1958-day EH2/EH1 and EH3/EH1 spectral ratios [53], 52 data points.

- Reno 2908-day FD/ND spectral ratio [15], 45 data points. 


\section{Accelerator experiments}

- MINOS $10.71 \times 10^{20}$ pot $\nu_{\mu}$-disappearance data [54], 39 data points.

- MINOS $3.36 \times 10^{20}$ pot $\bar{\nu}_{\mu}$-disappearance data [54], 14 data points.

- MINOS $10.6 \times 10^{20}$ pot $\nu_{e}$-appearance data [55], 5 data points.

- MINOS $3.3 \times 10^{20}$ pot $\bar{\nu}_{e}$-appearance data [55], 5 data points.

- T2K $19.7 \times 10^{20}$ pot $\nu_{\mu}$-disappearance data [8], 35 data points.

- T2K $19.7 \times 10^{20}$ pot $\nu_{e}$-appearance data [8], 23 data points for the CCQE and 16 data points for the $\mathrm{CC} 1 \pi$ samples.

- T2K $16.3 \times 10^{20}$ pot $\bar{\nu}_{\mu}$-disappearance data [8], 35 data points.

- T2K $16.3 \times 10^{20}$ pot $\bar{\nu}_{e}$-appearance data [8], 23 data points.

- NOvA $13.6 \times 10^{20}$ pot $\nu_{\mu}$-disappearance data [10], 76 data points.

- NOvA $13.6 \times 10^{20}$ pot $\nu_{e}$-appearance data [10], 13 data points.

- NOvA $12.5 \times 10^{20}$ pot $\bar{\nu}_{\mu}$-disappearance data [10], 76 data points.

- NOvA $12.5 \times 10^{20}$ pot $\bar{\nu}_{e}$-appearance data [10], 13 data points.

Open Access. This article is distributed under the terms of the Creative Commons Attribution License (CC-BY 4.0), which permits any use, distribution and reproduction in any medium, provided the original author(s) and source are credited.

\section{References}

[1] I. Esteban, M.C. Gonzalez-Garcia, M. Maltoni, I. Martinez-Soler and T. Schwetz, Updated fit to three neutrino mixing: exploring the accelerator-reactor complementarity, JHEP 01 (2017) 087 [arXiv: 1611.01514] [INSPIRE].

[2] I. Esteban, M.C. Gonzalez-Garcia, A. Hernandez-Cabezudo, M. Maltoni and T. Schwetz, Global analysis of three-flavour neutrino oscillations: synergies and tensions in the determination of $\theta_{23}, \delta_{C P}$, and the mass ordering, JHEP 01 (2019) 106 [arXiv:1811.05487] [INSPIRE].

[3] P.F. de Salas et al., 2020 Global reassessment of the neutrino oscillation picture, arXiv:2006.11237 [INSPIRE].

[4] P.F. De Salas, S. Gariazzo, O. Mena, C.A. Ternes and M. Tórtola, Neutrino Mass Ordering from Oscillations and Beyond: 2018 Status and Future Prospects, Front. Astron. Space Sci. 5 (2018) 36 [arXiv: 1806.11051] [INSPIRE].

[5] F. Capozzi, E. Di Valentino, E. Lisi, A. Marrone, A. Melchiorri and A. Palazzo, Global constraints on absolute neutrino masses and their ordering, Phys. Rev. D 95 (2017) 096014 [Addendum ibid. 101 (2020) 116013] [arXiv: 2003.08511] [INSPIRE].

[6] F. Capozzi, E. Lisi, A. Marrone and A. Palazzo, Current unknowns in the three neutrino framework, Prog. Part. Nucl. Phys. 102 (2018) 48 [arXiv:1804.09678] [inSPIRE]. 
[7] T2K collaboration, Constraint on the matter-antimatter symmetry-violating phase in neutrino oscillations, Nature 580 (2020) 339 [Erratum ibid. 583 (2020) E16] [arXiv: 1910.03887] [INSPIRE].

[8] P. Dunne, Latest Neutrino Oscillation Results from T2K, talk given at the XXIX International Conference on Neutrino Physics and Astrophysics, Chicago, U.S.A., June 22-July 2, 2020 (online conference) doi.org/10.5281/zenodo.3959558.

[9] NOvA collaboration, First Measurement of Neutrino Oscillation Parameters using Neutrinos and Antineutrinos by NOvA, Phys. Rev. Lett. 123 (2019) 151803 [arXiv: 1906.04907] [INSPIRE].

[10] A. Himmel, New Oscillation Results from the NOvA Experiment, talk given at the XXIX International Conference on Neutrino Physics and Astrophysics, Chicago, U.S.A., June 22-July 2, 2020 (online conference) doi.org/10.5281/zenodo.3959581.

[11] NuFit webpage, http://www.nu-fit.org.

[12] Double CHOOZ collaboration, Double CHOOZ $\theta_{13}$ measurement via total neutron capture detection, Nature Phys. 16 (2020) 558 [arXiv:1901.09445] [INSPIRE].

[13] T. Bezerra, New Results from the Double Chooz Experiment, talk given at the XXIX International Conference on Neutrino Physics and Astrophysics, Chicago, U.S.A., June 22-July 2, 2020 (online conference) doi.org/10.5281/zenodo.3959542.

[14] RENO collaboration, Measurement of Reactor Antineutrino Oscillation Amplitude and Frequency at RENO, Phys. Rev. Lett. 121 (2018) 201801 [arXiv:1806.00248] [INSPIRE].

[15] J. Yoo, RENO, talk given at the XXIX International Conference on Neutrino Physics and Astrophysics, Chicago, U.S.A., June 22-July 2, 2020 (online conference) doi.org/10.5281/zenodo.3959698.

[16] Y. Nakajima, SuperKamiokande, talk given at the XXIX International Conference on Neutrino Physics and Astrophysics, Chicago, U.S.A., June 22-July 2, 2020 (online conference) doi.org/10.5281/zenodo.3959640.

[17] M.C. Gonzalez-Garcia, M. Maltoni, J. Salvado and T. Schwetz, Global fit to three neutrino mixing: critical look at present precision, JHEP 12 (2012) 123 [arXiv: 1209.3023] [INSPIRE].

[18] M.C. Gonzalez-Garcia, M. Maltoni and T. Schwetz, Updated fit to three neutrino mixing: status of leptonic CP-violation, JHEP 11 (2014) 052 [arXiv:1409.5439] [INSPIRE].

[19] J. Elevant and T. Schwetz, On the determination of the leptonic CP phase, JHEP 09 (2015) 016 [arXiv: 1506.07685] [INSPIRE].

[20] K.J. Kelly, P.A. Machado, S.J. Parke, Y.F. Perez Gonzalez and R. Zukanovich-Funchal, Back to (Mass-)Square(d) One: The Neutrino Mass Ordering in Light of Recent Data, arXiv:2007.08526 [INSPIRE].

[21] L. Wolfenstein, Neutrino Oscillations in Matter, Phys. Rev. D 17 (1978) 2369 [INSPIRE].

[22] H. Nunokawa, S.J. Parke and R. Zukanovich Funchal, Another possible way to determine the neutrino mass hierarchy, Phys. Rev. D 72 (2005) 013009 [hep-ph/0503283] [INSPIRE].

[23] H. Minakata, H. Nunokawa, S.J. Parke and R. Zukanovich Funchal, Determining neutrino mass hierarchy by precision measurements in electron and muon neutrino disappearance experiments, Phys. Rev. D 74 (2006) 053008 [hep-ph/0607284] [InSPIRE].

[24] M. Maltoni and T. Schwetz, Testing the statistical compatibility of independent data sets, Phys. Rev. D 68 (2003) 033020 [hep-ph/0304176] [InSPIRE].

[25] S.P. Mikheyev and A. Smirnov, Resonance Amplification of Oscillations in Matter and Spectroscopy of Solar Neutrinos, Sov. J. Nucl. Phys. 42 (1985) 913 [INSPIRE]. 
[26] M. Ikeda, Solar neutrino measurements with Super-Kamiokande, talk given at the XXVIII International Conference on Neutrino Physics and Astrophysics, Heidelberg, Germany, June $4-9,2018$.

[27] Y. Nakano, ${ }^{8}$ B solar neutrino spectrum measurement using Super-Kamiokande IV, PhD thesis, Tokyo University (February 2016).

[28] BOREXINO collaboration, First Direct Experimental Evidence of CNO neutrinos, arXiv:2006.15115 [INSPIRE].

[29] J. Bergstrom, M.C. Gonzalez-Garcia, M. Maltoni, C. Pena-Garay, A.M. Serenelli and N. Song, Updated determination of the solar neutrino fluxes from solar neutrino data, JHEP 03 (2016) 132 [arXiv: 1601.00972] [INSPIRE].

[30] N. Vinyoles et al., A new Generation of Standard Solar Models, Astrophys. J. 835 (2017) 202 [arXiv: 1611.09867] [INSPIRE].

[31] SuperKamiokAnde collaboration, Atmospheric neutrino oscillation analysis with external constraints in Super-Kamiokande I-IV, 2018, link to data release: http://www-sk.icrr.u-tokyo.ac.jp/sk/publications/result-e.html\#atmosci2018.

[32] Super-Kamiokande collaboration, Atmospheric Neutrino Oscillation Analysis with Improved Event Reconstruction in Super-Kamiokande IV, PTEP 2019 (2019) 053F01 [arXiv: 1901.03230] [INSPIRE].

[33] M.C. Gonzalez-Garcia and C. Pena-Garay, Three neutrino mixing after the first results from K2K and KamLAND, Phys. Rev. D 68 (2003) 093003 [hep-ph/0306001] [INSPIRE].

[34] P.I. Krastev and S.T. Petcov, Resonance Amplification and t Violation Effects in Three Neutrino Oscillations in the Earth, Phys. Lett. B 205 (1988) 84 [InSPIRE].

[35] C. Jarlskog, Commutator of the Quark Mass Matrices in the Standard Electroweak Model and a Measure of Maximal CP-violation, Phys. Rev. Lett. 55 (1985) 1039 [InSPIRE].

[36] Particle Data Group collaboration, Review of Particle Physics, Phys. Rev. D 98 (2018) 030001 [INSPIRE].

[37] B.T. Cleveland et al., Measurement of the solar electron neutrino flux with the Homestake chlorine detector, Astrophys. J. 496 (1998) 505 [INSPIRE].

[38] F. Kaether, W. Hampel, G. Heusser, J. Kiko and T. Kirsten, Reanalysis of the GALLEX solar neutrino flux and source experiments, Phys. Lett. B 685 (2010) 47 [arXiv:1001.2731] [INSPIRE].

[39] SAGE collaboration, Measurement of the solar neutrino capture rate with gallium metal. III: Results for the 2002-2007 data-taking period, Phys. Rev. C 80 (2009) 015807 [arXiv:0901.2200] [INSPIRE].

[40] Super-KAmioKAnde collaboration, Solar neutrino measurements in Super-Kamiokande-I, Phys. Rev. D 73 (2006) 112001 [hep-ex/0508053] [INSPIRE].

[41] Super-KamiokAnde collaboration, Solar neutrino measurements in Super-Kamiokande-II, Phys. Rev. D 78 (2008) 032002 [arXiv:0803.4312] [INSPIRE].

[42] Super-Kamiokande collaboration, Solar neutrino results in Super-Kamiokande-III, Phys. Rev. D 83 (2011) 052010 [arXiv:1010.0118] [INSPIRE].

[43] SNO collaboration, Combined Analysis of all Three Phases of Solar Neutrino Data from the Sudbury Neutrino Observatory, Phys. Rev. C 88 (2013) 025501 [arXiv:1109.0763] [INSPIRE].

[44] G. Bellini et al., Precision measurement of the 7Be solar neutrino interaction rate in Borexino, Phys. Rev. Lett. 107 (2011) 141302 [arXiv:1104.1816] [INSPIRE]. 
[45] Borexino collaboration, Measurement of the solar $8 B$ neutrino rate with a liquid scintillator target and $3 \mathrm{MeV}$ energy threshold in the Borexino detector, Phys. Rev. D 82 (2010) 033006 [arXiv: 0808.2868] [INSPIRE].

[46] BOREXINO collaboration, Neutrinos from the primary proton-proton fusion process in the Sun, Nature 512 (2014) 383 [INSPIRE].

[47] M. Honda, M. Sajjad Athar, T. Kajita, K. Kasahara and S. Midorikawa, Atmospheric neutrino flux calculation using the NRLMSISE-00 atmospheric model, Phys. Rev. D 92 (2015) 023004 [arXiv: 1502.03916] [INSPIRE].

[48] ICECuBe collaboration, Determining neutrino oscillation parameters from atmospheric muon neutrino disappearance with three years of IceCube DeepCore data, Phys. Rev. D 91 (2015) 072004 [arXiv:1410.7227] [INSPIRE].

[49] ICECUBE collaboration, IceCube Oscillations: 3 years muon neutrino disappearance data, http://icecube.wisc.edu/science/data/nu_osc.

[50] Super-Kamiokande collaboration, Atmospheric neutrino oscillation analysis with external constraints in Super-Kamiokande I-IV, Phys. Rev. D 97 (2018) 072001 [arXiv:1710.09126] [INSPIRE].

[51] KamLAND collaboration, Reactor On-Off Antineutrino Measurement with KamLAND, Phys. Rev. D 88 (2013) 033001 [arXiv:1303.4667] [InSPIRE].

[52] DaYA BAY collaboration, Improved Measurement of the Reactor Antineutrino Flux and Spectrum at Daya Bay, Chin. Phys. C 41 (2017) 013002 [arXiv: 1607.05378] [INSPIRE].

[53] DaYa Bay collaboration, Measurement of the Electron Antineutrino Oscillation with 1958 Days of Operation at Daya Bay, Phys. Rev. Lett. 121 (2018) 241805 [arXiv:1809.02261] [INSPIRE].

[54] MINOS collaboration, Measurement of Neutrino and Antineutrino Oscillations Using Beam and Atmospheric Data in MINOS, Phys. Rev. Lett. 110 (2013) 251801 [arXiv:1304.6335] [INSPIRE].

[55] MINOS collaboration, Electron neutrino and antineutrino appearance in the full MINOS data sample, Phys. Rev. Lett. 110 (2013) 171801 [arXiv:1301.4581] [INSPIRE]. 